\title{
Cuantificación del consumo de gases anestésicos y medicinales después de la implementación de anestesia general a bajo flujo
}

\author{
Matías Ramos*, Franco Fratebianchi, Josefina Lafourcade, Bruno Santoro, María J. Laiño y \\ Diego S. Guardabassi
}

Servicio de Anestesiología Hospital de Clínicas, Asociación de Anestesia, Analgesia y Reanimación de Buenos Aires (AAARBA), Buenos Aires, Argentina

\section{Resumen}

Objetivos: Relevar el consumo de gases anestésicos y medicinales y compararlo de acuerdo a si se utilizó o no anestesia a bajo flujo, asimismo, estimar el consumo de oxígeno si las anestesias generales se hubieran realizado con estaciones de trabajo con concertina. Material y métodos: Se obtuvieron los datos de consumo en diferentes cirugías, comparándose estos con el mismo período del año anterior, momento en el que no se realizaba anestesia a bajo flujo. También se estimó cómo hubiese aumentado el consumo de oxígeno si las mismas cirugías relevadas se hubieran efectuado con una máquina de anestesia con tecnología de concertina. Resultados: La recolección de datos incluyó 59 anestesias generales. La cantidad de anestesias por frasco de sevoflurano aumentó de 12.1 a 18.7 después de la implementación parcial de la técnica de bajo flujo. Se estimó un ahorro de 45,633 I de oxígeno al utilizar pistón. Discusión: La precisión en el aporte de gas fresco y el análisis de gases espirados parece reducir el consumo de agente anestésico. Se halló que la eficiencia en el uso de sevoflurano no es óptima, y que la utilización sistemática de la técnica de bajo flujo aportaría beneficios para el paciente, el medio ambiente, la salud del personal de quirófano y la economía institucional.

Palabras clave: Consumo anestésico. Costo-efectividad. Anestesia a bajo flujo. Anestésicos volátiles.

\section{Quantification of anesthetic and medicinal gas consumption after the implementation of general low-flow anesthesia}

\begin{abstract}
Objectives: Relieve the consumption of anesthetic agent and medical gases and compare those according to whether lowflow anesthesia was used or not, and likewise estimate oxygen consumption if general anesthesia had been performed with workstations with bellows. Material and methods: Data on the consumption of anesthetic and medicinal gases in different surgeries were obtained, comparing those with the same period of the previous year, when low-flow anesthesia was not performed. In addition, we estimated how oxygen consumption would have increased if the same surgeries had been performed with an anesthesia machine with bellows technology. Results: Data collection included 59 general anesthesias. The
\end{abstract}

Correspondencia:

*Matías Ramos

E-mail: matt.plate@gmail.com
Fecha de recepción: 26-04-2019

Fecha de aceptación: 11-09-2019

DOI: 10.24875/RAA.19000031
Disponible en internet: 22-11-2019 Rev Argent Anestesiol. 2019;77(1):6-12 www. revistaargentinaanestesiologia.com 0370-7792 @ 2019 Federación Argentina de Asociaciones, Anestesia, Analgesia y Reanimación. Publicado por Permanyer México SA de CV. Este es un artículo open access bajo la licencia CC BY-NC-ND (http://creativecommons.org/licenses/by-nc-nd/4.0/). 
amount of procedures per sevoflurane bottle increased from 12.1 to 18.7 after partial implementation of low-flow technique. Savings of 45,633 L in oxygen were estimated when using piston technology in an anesthesia workstation. Discussion: The accuracy in fresh gas delivery and the analysis of expired gases seems to reduce the anesthetic agent consumption. It was found that the efficiency in the use of sevoflurane is not optimal and the systematic use of low-flow technique would bring benefits not only for the patient, but for the environment, the health of the operating room staff and the institutional economy.

Key words: Anesthetic consumption. Cost-effectiveness. Low-flow anesthesia. Volatile anesthetics.

\section{Introducción}

Con el progreso tecnológico, las estaciones de trabajo para anestesia han evolucionado y cuentan con una tecnología que suma funciones y mejora el cuidado del paciente. Con sofisticados sistemas de monitoreo, precisos vaporizadores de agentes anestésicos, modernos modos de ventilación mecánica y grandes monitores que facilitan la entrega de información, el anestesiólogo se ve en la obligación de entender los principios de funcionamiento del equipamiento para así controlar formas de anestesia, como la anestesia a bajo flujo, que sin la tecnología actual sería impensable.

En referencia a esto último, las modernas estaciones de trabajo permiten un monitoreo preciso de los gases proporcionados al paciente bajo anestesia general y una cuantificación precisa, por medio de flujímetros electrónicos, del aporte de gas fresco al circuito anestésico. Tanto el valor de flujo de gas fresco (FGF) como el porcentaje configurado en el vaporizador son las variables que determinan el aporte de agente halogenado al sistema.

Teniendo en cuenta la importancia de los gastos en salud, trabajos previos ${ }^{1}$ muestran que tanto la anestesia a bajo flujo, como el empleo de la tecnología de pistón en el ventilador mecánico, conllevan al ahorro, por un lado de agente anestésico, al disminuir los desechos que abandonan el circuito por el sistema de eliminación, y por otro, de gases medicinales, al no utilizar gas propulsor para movilizar la concertina.

Las bases de la anestesia a bajo flujo fueron fundadas por Lowe y Ernst en $1981^{2}$, al introducir el concepto de «circuito cerrado" en la práctica anestésica. Esta técnica provee ventajas ${ }^{3}$ al paciente: incrementa el aclaramiento mucociliar, conserva la temperatura y reduce la pérdida de humedad; al ambiente: disminuye la emisión de gases anestésicos, con lo que logra un quirófano más seguro y evita el impacto sobre la capa de ozono; y a la economía institucional: ahorro de gases anestésicos y medicinales ${ }^{4,5}$. La anestesia a bajo flujo puede clasificarse de acuerdo a la cantidad de FGF empleado en: bajo flujo propiamente dicho (1 l/min), flujo mínimo (0.5 l/min) y flujo metabólico $(0.35 \mathrm{l} / \mathrm{min})$. Esta técnica se posiciona como un procedimiento económico, eficiente, elegante y sobre todo seguro para nuestros pacientes ${ }^{6}$.

Por otro lado, si la tecnología utilizada en el ventilador mecánico de la estación de trabajo es el pistón, se acarrean otro tipo de ventajas al compararse con la concertina: no se utiliza gas motriz, lo que implica ahorro de oxígeno, y se entrega con mayor precisión el volumen corriente configurado ${ }^{7}$. Tanto la anestesia a bajo flujo, como el uso del pistón en el ventilador mecánico, son tecnologías que proveen un potencial ahorro en anestesia.

El objetivo del presente estudio fue evaluar el consumo del agente sevoflurano y de gases medicinales (oxígeno y aire) por el Servicio de Anestesia en los quirófanos centrales del Hospital de Clínicas durante el periodo comprendido entre el 6 de agosto y el 3 de septiembre de 2018 en pacientes bajo anestesia general. También se comparó con el consumo de sevoflurano del año anterior, momento en el cual no se realizaba anestesia a bajo flujo y la tecnología para entregar el volumen minuto al paciente bajo anestesia era por medio de concertina propulsada por gases medicinales.

\section{Material y métodos}

Una vez aprobado el protocolo por el Servicio de Anestesiología del Hospital de Clínicas, los datos fueron recolectados de las estaciones de trabajo y volcados en un registro electrónico. Las variables que fueron incorporadas en el registro fueron: tipo de cirugía, duración del procedimiento (variable proporcionada por la estación de trabajo, desde que se apoya la máscara facial para preoxigenar al paciente hasta que se realiza la extubación), consumo de sevoflurano (volumen aportado al circuito), captación de sevoflurano (volumen captado por el paciente), eficiencia del procedimiento (entendida como la relación captación/consumo), consumo de gases medicinales (volumen de aire y oxígeno utilizados) y estimación del consumo extra de oxígeno si la cirugía se hubiese realizado con concertina como método para la entrega del volumen corriente. Esta estimación se hace con base en que el volumen de gas propulsor que utiliza la concertina es igual al volumen 
minuto respiratorio del paciente. Se estimó una frecuencia promedio de 12 ventilaciones/minuto y un volumen corriente de $400 \mathrm{ml}$. Al multiplicar el volumen minuto respiratorio estimado por la duración de la cirugía, se obtiene un valor estimado del gas motriz que se hubiese usado. El «consumo» y la "captación» de sevoflurano son calculados automáticamente por la estación de trabajo Drager Primus ${ }^{8}$ al finalizar el procedimiento en el apartado «Diario» de la interfaz.

Por medio de un sistema informático se aleatorizó a los pacientes por el método de clústeres (cirugías consecutivas tal como figuran en la lista quirúrgica provista por la Jefatura de Departamento Quirúrgico, realizadas en un quirófano para cada 3 días hábiles). Se incluyeron pacientes adultos, bajo anestesia general, cuya extubación se produjera en quirófano. No hubo restricciones sobre el tipo o la duración de la cirugía.

Por otro lado, por medio de farmacia central, se obtuvo la cantidad de frascos de sevoflurano comprados mensualmente en el periodo de julio - agosto de 2017 y 2018, así como su precio actual.

El análisis estadístico fue realizado utilizando un programa estándar (GraphPad Software). La normalidad de los datos fue analizada mediante el test de WiIk-Shapiro. Los datos normalmente distribuidos están presentados como media \pm desviación estándar, los no-normalmente distribuidos como mediana (rango) y los categóricos como data cruda y frecuencias.

\section{Resultados}

En la tabla 1 se resumen los datos obtenidos. Se recolectaron datos de 59 procedimientos bajo anestesia general. La duración promedio fue de 161 minutos/procedimiento (60 - $683 \mathrm{~min}$ ). El consumo de oxígeno promedio fue de $231 \mathrm{l} /$ procedimiento (52 - 856 l) y el consumo de aire 87 I (18 - 349 I). El consumo de sevoflurano promedio fue de $18.1 \mathrm{ml} /$ procedimiento (4 - $68 \mathrm{ml} /$ procedimiento), la captación de sevoflurano promedio fue de $10.1 \mathrm{ml} /$ procedimiento $(3-43 \mathrm{ml} /$ procedimiento), la eficiencia promedio en el uso fue de $60 \%$ (20 - 100\%). Por último, la estimación del consumo extra de gas motriz fue de 773.45 I por cirugía $(288-3,276 \mathrm{I})$.

En el periodo de julio/agosto de 2017 se compraron 69 frascos de $250 \mathrm{ml}$ de sevoflurano, mientras que en 2018 se compraron 57. En el mismo periodo de 2017 se realizaron 838 anestesias generales en quirófano central, lo que da un promedio de 12.1 anestesias/ frasco. En 2018 se realizaron 1,070 anestesias generales, con un promedio de 18.7 anestesias/frasco. Los datos muestran un ahorro de 0.029 frascos por cirugía. A un precio actual de US\$ 94.2 por botella, el ahorro es de US\$2.7 por cirugía.

Por otro lado, el consumo de oxígeno total en las cirugías registradas fue de 13,648 I. Si las mismas cirugías se hubiesen realizado con tecnología de concertina, se hubiesen consumido 59,281 I, lo que muestra un ahorro del $334.3 \%$, con un promedio de 773 । de oxígeno ahorrados por anestesia general.

\section{Discusión}

Como se ha demostrado previamente ${ }^{9}$, nuestros datos indican que la cuantificación precisa del aporte de gas fresco y el análisis de gases por parte de la estación de trabajo, sin otra intervención, provocan una reducción en el consumo total de gases anestésicos. Esto es posible ya que, al contar con retroalimentación sobre la concentración alveolar del agente, consumo de oxígeno del paciente, cuantificación en tiempo real de la fuga del sistema, análisis de los gases espirados y precisión en la configuración del FGF, el anestesiólogo cuenta con niveles de seguridad adecuados para reducir el aporte de gas fresco al sistema.

Con los datos obtenidos podemos observar la relación entre el consumo de sevoflurano y la duración de la anestesia (Fig. 1), y se pone en evidencia que el consumo es muy dispar: hay anestesias generales de menor duración donde se consume más agente que en procedimientos de mayor duración. Desde un análisis costo-efectivo, podemos afirmar que en determinadas anestesias generales hay desperdicio de gases, que perfeccionando la técnica de bajo flujo, podría evitarse.

Entendemos que la familiarización con el bajo flujo tiene un curva de aprendizaje para el anestesiólogo, y que si se conocen sus precauciones, se puede lograr una anestesia más sustentable, prolija y eficiente, sin comprometer, en absoluto, la seguridad del paciente. Dentro de sus limitaciones, cuando esta técnica se implementa se deben configurar alarmas de seguridad para evitar generar mezclas hipóxicas para el paciente (cuando el aporte de oxígeno es menor que el consumo del paciente), controlar fugas que despresuricen el circuito y tener un sistema eficiente de eliminación de dióxido de carbono. Si bien el consumo de cal sodada en bajo flujo es mayor que cuando se utilizan flujos de gas fresco altos, al compararse con el ahorro de sevoflurano, los gastos son despreciables en el análisis de rentabilidad total ${ }^{6}$. Por otro lado, en determinadas condiciones clínicas, como la cetoacidosis diabética o la 
Tabla 1. Cirugías registradas

\begin{tabular}{|c|c|c|c|c|c|c|c|}
\hline Cirugía & $\begin{array}{l}\text { Duración } \\
\text { (min) }\end{array}$ & $\begin{array}{l}\mathbf{O}_{2} \\
\text { (L) }\end{array}$ & $\begin{array}{l}\text { Aire } \\
\text { (L) }\end{array}$ & $\begin{array}{c}\text { Consumo } \\
\text { (mL) }\end{array}$ & $\begin{array}{l}\text { Captación } \\
\text { (mL) }\end{array}$ & $\begin{array}{c}\text { Eficiencia } \\
(\%)\end{array}$ & $\begin{array}{l}\text { Concertina } \\
\text { (L) }\end{array}$ \\
\hline Histerectomía & 98 & 140 & 69 & 20 & 7 & $35.0 \%$ & 470.4 \\
\hline Tiroidectomía & 163 & 266 & 91 & 26 & 15 & $57.7 \%$ & 782.4 \\
\hline Histerectomía + salpingectomía & 170 & 20 & 165 & 27 & 14 & $51.9 \%$ & 816 \\
\hline Nodulectomía múltiple & 76 & 172 & 81 & 10 & 6 & $60.0 \%$ & 364.8 \\
\hline Colecistectomía & 148 & 234 & 75 & 17 & 12 & $70.6 \%$ & 710.4 \\
\hline Bypass gástrico & 180 & 623 & 122 & 33 & 13 & $39.4 \%$ & 864 \\
\hline Dermolipectomía & 148 & 286 & 185 & 21 & 7 & $33.3 \%$ & 710.4 \\
\hline Artrodesis lumbar & 192 & 368 & 94 & 18 & 10 & $55.6 \%$ & 921.6 \\
\hline Cirugía comando & 683 & 427 & 235 & 59 & 43 & $72.9 \%$ & $3,278.4$ \\
\hline Retiro de prótesis mamarias & 138 & 209 & 102 & 14 & 5 & $35.7 \%$ & 662.4 \\
\hline Wertheim Meigs & 251 & 300 & 136 & 31 & 15 & $48.4 \%$ & $1,204.8$ \\
\hline Colecistectomía & 91 & 264 & 44 & 14 & 7 & $50.0 \%$ & 436.8 \\
\hline Bypass gástrico & 148 & 274 & 75 & 17 & 12 & $70.6 \%$ & 710.4 \\
\hline Gastroenteroanastomosis & 103 & 164 & 21 & 8 & 7 & $87.5 \%$ & 494.4 \\
\hline Parotidectomía & 240 & 344 & 126 & 20 & 8 & $40.0 \%$ & 1,152 \\
\hline Tiroidectomía & 190 & 242 & 162 & 27 & 12 & $44.4 \%$ & 912 \\
\hline Colecistectomía & 140 & 144 & 35 & 9 & 8 & $88.9 \%$ & 672 \\
\hline Safenectomía & 143 & 257 & 72 & 12 & 8 & $66.7 \%$ & 686.4 \\
\hline Colecistectomía & 123 & 218 & 80 & 9 & 4 & $44.4 \%$ & 590.4 \\
\hline Herniplastia umbilical & 69 & 95 & 47 & 15 & 4 & $26.7 \%$ & 331.2 \\
\hline Adenomastectomía bilateral & 307 & 226 & 169 & 29 & 19 & $65.5 \%$ & $1,473.6$ \\
\hline Toilette abdominal & 153 & 75 & 42 & 10 & 7 & $70.0 \%$ & 734.4 \\
\hline Adenomastectomía bilateral & 64 & 125 & 56 & 15 & 3 & $20.0 \%$ & 307.2 \\
\hline Anexohisterectomía laparoscópica & 132 & 144 & 30 & 11 & 7 & $63.6 \%$ & 633.6 \\
\hline Salpingectomía laparoscópica & 95 & 217 & 60 & 11 & 8 & $72.7 \%$ & 456 \\
\hline Toilette de columna & 146 & 240 & 88 & 11 & 7 & $63.6 \%$ & 700.8 \\
\hline Vaciamiento ganglionar cervical & 91 & 293 & 31 & 9 & 7 & $77.8 \%$ & 436.8 \\
\hline Vulvectomía simple & 70 & 145 & 35 & 8 & 5 & $62.5 \%$ & 336 \\
\hline Cuadrantectomía mamaria & 89 & 149 & 47 & 14 & 5 & $35.7 \%$ & 427.2 \\
\hline Blefaroplastia bilateral & 191 & 276 & 145 & 25 & 14 & $56.0 \%$ & 916.8 \\
\hline Escisión de tumor de piel & 172 & 334 & 152 & 35 & 16 & $45.7 \%$ & 825.6 \\
\hline Hernioplastia hiatal & 426 & 856 & 122 & 68 & 25 & $36.8 \%$ & $2,044.8$ \\
\hline Escisión de tumor de piel & 91 & 293 & 31 & 9 & 7 & $77.8 \%$ & 436.8 \\
\hline Gangrena de Fournier & 73 & 142 & 19 & 4 & 3 & $75.0 \%$ & 350.4 \\
\hline Embarazo ectópico & 102 & 128 & 21 & 7 & 6 & $85.7 \%$ & 489.6 \\
\hline Escisión de tumor de piel & 80 & 161 & 41 & 8 & 5 & $62.5 \%$ & 384 \\
\hline
\end{tabular}


Rev Argent Anestesiol. 2019;77

Tabla 1. Cirugías registradas (Continuación)

\begin{tabular}{|c|c|c|c|c|c|c|c|}
\hline Cirugía & $\begin{array}{l}\text { Duración } \\
\text { (min) }\end{array}$ & $\begin{array}{l}\mathbf{O}_{2} \\
\text { (L) }\end{array}$ & $\begin{array}{c}\text { Aire } \\
\text { (L) }\end{array}$ & $\begin{array}{c}\text { Consumo } \\
\text { (mL) }\end{array}$ & $\begin{array}{c}\text { Captación } \\
(\mathrm{mL})\end{array}$ & $\begin{array}{c}\text { Eficiencia } \\
(\%)\end{array}$ & $\begin{array}{l}\text { Concertina } \\
\text { (L) }\end{array}$ \\
\hline Mastoplastia bilateral & 182 & 191 & 131 & 18 & 10 & $55.6 \%$ & 873.6 \\
\hline Recambio expansor mamario & 75 & 146 & 39 & 10 & 6 & $60.0 \%$ & 360 \\
\hline Reemplazo total de cadera & 286 & 259 & 130 & 31 & 14 & $45.2 \%$ & $1,372.8$ \\
\hline Artrodesis de cadera & 191 & 276 & 88 & 21 & 13 & $61.9 \%$ & 916.8 \\
\hline Colecistectomía & 108 & 120 & 25 & 7 & 6 & $85.7 \%$ & 518.4 \\
\hline Biopsia de columna & 60 & 197 & 45 & 6 & 4 & $66.7 \%$ & 288 \\
\hline Nefrolitotricia percutánea & 130 & 182 & 59 & 15 & 10 & $66.7 \%$ & 624 \\
\hline Nefrolitotricia percutánea & 170 & 210 & 42 & 15 & 11 & $73.3 \%$ & 816 \\
\hline Reconstrucción tránsito intestinal & 180 & 158 & 65 & 15 & 12 & $80.0 \%$ & 864 \\
\hline Laparotomía exploradora & 301 & 217 & 349 & 49 & 30 & $61.2 \%$ & $1,444.8$ \\
\hline Colecistectomía & 104 & 124 & 123 & 13 & 8 & $61.5 \%$ & 499.2 \\
\hline Laparotomía exploradora & 141 & 100 & 67 & 9 & 6 & $66.7 \%$ & 676.8 \\
\hline Hernioplastia inguinal & 74 & 52 & 33 & 4 & 3 & $75.0 \%$ & 355.2 \\
\hline Tenorrafia & 130 & 133 & 18 & 9 & 7 & $77.8 \%$ & 624 \\
\hline Cifoplastia lumbar & 115 & 398 & 43 & 27 & 8 & $29.6 \%$ & 552 \\
\hline Perforación intestinal & 295 & 306 & 60 & 14 & 11 & $78.6 \%$ & 1,416 \\
\hline Rinoplastia & 140 & 199 & 99 & 11 & 6 & $54.5 \%$ & 672 \\
\hline Linfadenectomía retroperitoneal & 260 & 422 & 177 & 42 & 31 & $73.8 \%$ & 1,248 \\
\hline Laparotomía exploradora & 230 & 122 & 36 & 14 & 12 & $85.7 \%$ & 1,104 \\
\hline Hemitiroidectomía & 96 & 158 & 137 & 11 & 4 & $36.4 \%$ & 460.8 \\
\hline Colecistectomía & 106 & 100 & 19 & 6 & 6 & $100.0 \%$ & 508.8 \\
\hline Histerectomía & 177 & 177 & 114 & 22 & 13 & $59.1 \%$ & 849.6 \\
\hline Parotidectomía & 180 & 350 & 168 & 22 & 9 & $40.9 \%$ & 864 \\
\hline
\end{tabular}

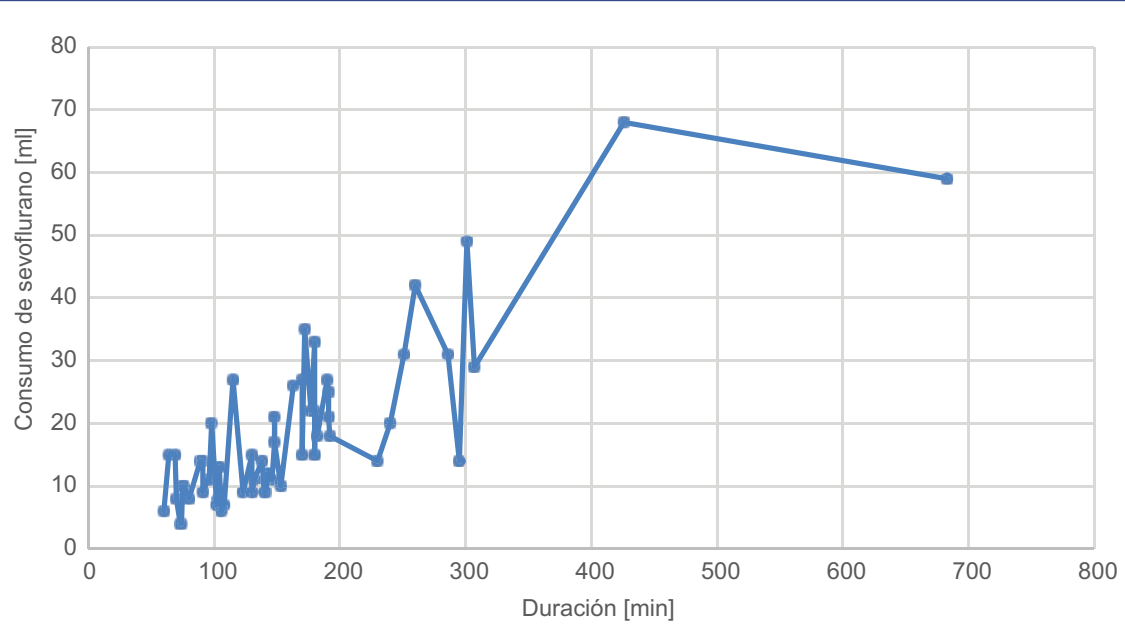

Figura 1. Relación entre el consumo de sevoflurano y la duración de la anestesia. 
intoxicación etílica, donde el paciente elimina tóxicos por vía respiratoria, la anestesia a bajo flujo podría tener un papel nocivo al permitir la reinhalación de dichos desechos.

Por otro lado, en términos de la distribución de la eficiencia, la mayor parte de los procedimientos se ubican en el rango del $50 \%$ al $75 \%$ (Fig. 2), lo que pone en evidencia una potencial mejora en este aspecto. Creemos que si la anestesia a bajo flujo se realizara en forma sistemática y protocolizada, la eficiencia mejoraría y los beneficios serían mayores a los obtenidos. Por ejemplo, si todas las anestesias generales registradas se hubieran realizado con una eficiencia promedio de los cinco procedimientos con mejor eficiencia (es decir, un estimado de $89.5 \%$ ), la cantidad de frascos de sevoflurano consumido en las cirugías registradas hubiese disminuido de 4.28 a 2.68 (una reducción del $59 \%$ ), lo que se traduce en un ahorro de US\$ 149.8 .

Desde otro punto de vista, los quirófanos que no cuenten con un sistema de recolección de gases residuales hacia el exterior, la misma técnica que permite ahorrar gases y abaratar costos, al reducir los desperdicios, evita una contaminación excesiva del quirófano ${ }^{10}$ con los fármacos que son administrados al paciente, lo que aumenta la seguridad del personal y contribuye a disminuir el impacto ambiental de la anestesia.

Por otro lado, las estaciones de trabajo concertina utilizan oxígeno como gas motriz para propulsarse. Esto es así por seguridad, ya que de existir una fuga en la concertina, el gas que se introduce al circuito es oxígeno medicinal. Este mecanismo de seguridad genera un gasto de gases medicinales adicional, independiente a si se utiliza o no bajo flujo. A este respecto, si bien los datos fueron estimados y no medidos, el análisis nos muestra que la tecnología de pistón en una estación de trabajo, disminuye considerablemente el consumo de gases medicinales.

Somos conscientes de la importancia del uso eficiente de los recursos que nuestro sistema de salud aporta a las instituciones con el fin de maximizar los resultados con el menor gasto posible. En este sentido, si bien el ahorro parece poco en el contexto del gasto hospitalario total, incluso pequeños números pueden hacer la diferencia si se multiplican por la cantidad de anestesias generales que se realizan. Sostenemos que los hallazgos de este trabajo pueden aportar datos importantes sobre esta temática, e impactar potencialmente en el uso de recursos en la salud pública ${ }^{11}$.

Forma parte de las limitaciones de nuestro trabajo el hecho de que se midió el consumo de gases medicinales incluso durante la inducción y la emergencia de

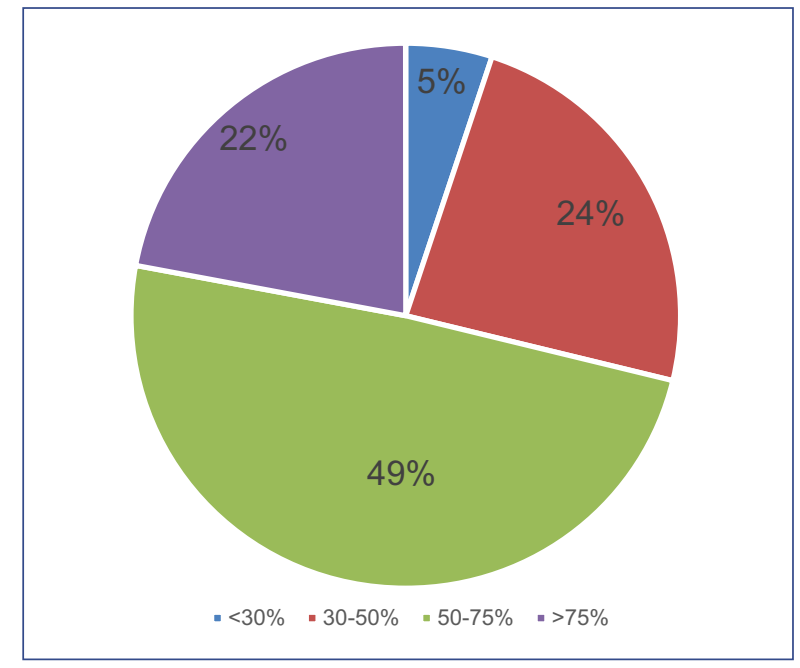

Figura 2. Distribución de la eficiencia en las cirugías registradas.

la anestesia general, momento en el cual las necesidades de flujo de gas fresco son mayores, ya sea para titular de forma más rápida y precisa el nivel de agente anestésico o para retirarlo del sistema de manera más veloz, respectivamente. Esto implica que no podemos evaluar el valor promedio de FGF que se usó en el mantenimiento de cada anestesia. También puede existir un sesgo al asumir un sistema regular de compras por parte de la farmacia del hospital. En el análisis de los datos se asumió que la compra de sevoflurano guarda relación con el consumo. Por otro lado, la reducción del consumo de gases medicinales (independientemente al uso de bajo flujo) al utilizar tecnología de pistón no fue medido, sino estimado, lo que aporta información semicuantitativa sobre el ahorro en este aspecto. Por último, otra limitante a tener en cuenta, es que el diseño de nuestra investigación es descriptivo, hecho que debe ser valorado a la hora de analizar nuestros hallazgos.

Consideramos que futuros ensayos, de tipo analítico experimental comparando ambas técnicas, multicéntricos y con un tamaño muestral adecuado, permitirán obtener conclusiones con un grado de evidencia más sólido. La presente investigación constituye un estudio piloto de relevancia y referencia para los mismos.

\section{Agradecimientos}

A todo el Servicio de Anestesiología del Hospital de Clínicas por facilitarnos los procesos para llevar a cabo la investigación. 


\section{Financiamiento}

Los autores declaran que no hubo financiamiento

\section{Conflicto de intereses}

Los autores declaran que no hay conflicto de intereses

\section{Responsabilidades éticas}

Protección de personas y animales. Los autores declaran que para esta investigación no se han realizado experimentos en seres humanos ni en animales.

Confidencialidad de los datos. Los autores declaran que han seguido los protocolos de su centro de trabajo sobre la publicación de datos de pacientes.

Derecho a la privacidad y consentimiento informado. Los autores declaran que en este artículo no aparecen datos de pacientes.

\section{Bibliografía}

1. Ryu H, Lee J, Lee K, Gil N, Kim CS, Sim S, et al. The effect of low fresh gas flow rate on sevoflurane consumption. Korean J Anesthesiol. 2011;60(2):75.

2. Lowe HJ, Ernst EA. The quantitative practice of anesthesia: use of closed circuits. Baltimore (MD): Williams \& Wilkins; 1981.

3. Hönemann C, Hagemann O, Doll D. Inhalational anaesthesia with low fresh gas flow. Indian J Anaesth. 2013;57(4):345.

4. Suttner S, Boldt J. Low-Flow Anaesthesia. Does it have potential pharmacoeconomic consequences? PharmacoEconomics. 2000;17(6):585-90.

5. Watcha MF, White P. Economics of anesthetic practice. Anesthesiology. 1997;86: 1170-96.

6. Hönemann C, Mierke B. Anestesia de flujo bajo, flujo mínimo y flujo metabólico Técnicas. Lübeck, Alemania: Drägerwerk AG \& Co. KGaA; 2016. Disponible en: https://www.draeger.com/Library/Content/Folleto\%20de\%20Anestesia\%20de\%20flujo\%20bajo.pdf

7. Divatia J, Patil V, Shetmahajan M. The modern integrated anaesthesia workstation. Indian J Anaesthesia. 2013:57(5):446.

8. Biro $P$, Kneschke O, Theusinger OM. Reliability of the volatile agent consumption display in the Draeger Primus ${ }^{\mathrm{TM}}$ anesthesia machine. J Clin Monit Comput. 2014;29(5):601-4.

9. Luis T, KaySee T, Austin H, Leslie O, Cindy Y. Analyzing Volatile Anesthetic Consumption by Auditing Fresh Gas Flow: An Observational Study at an Academic Hospital. Int J Anesth Anesthesiol. 2018;5(1):064

10. Molina Aragonés JM, Ayora AA, Ribalta AB, Aparici AG, Lavela JA, Vidiella JS, et al. Occupational exposure to volatile anaesthetics: a systematic review. Occupational Medicine. 2015;66(3),202-7.

11. Wetchler BV. Economic impact of anesthesia decision making: They pay the money, we make the choice. J Clin Anesth. 1992;4(5):20-4. 